\title{
Intuitiveness of Symbol Features for Air Traffic Management
}

\author{
Mary Kim Ngo ${ }^{1}$, Kim-Phuong L. Vu ${ }^{1}$, Elaine Thorpe ${ }^{1}$, Vernol Battiste ${ }^{2}, \&$ Thomas Z. Strybel ${ }^{1}$ \\ ${ }^{1}$ Center for Human Factors in Advanced Aeronautics Technologies (CHAAT) \\ California State University, Long Beach \\ ${ }^{2}$ Flight Deck Display Research Laboratory \\ NASA Ames Research Center
}

\begin{abstract}
We present the results of two online surveys asking participants to indicate what type of air traffic information might be conveyed by a number of symbols and symbol features (color, fill, text, and shape). The results of this initial study suggest that the well-developed concepts of ownership, altitude, and trajectory are readily associated with certain symbol features, while the relatively novel concept of equipage was not clearly associated with any specific symbol feature.
\end{abstract}

\section{INTRODUCTION}

The Next Generation Air Transportation System (NextGen) is an ongoing program of improvements and upgrades to the National Airspace System (NAS) for enhancing the safety, reliability, and efficiency of air transportation (FAA, 2011). To achieve these goals, all components of the NAS, including air traffic management operations, airports, air operations centers and flight decks are being overhauled. NextGen changes are being rolled out incrementally, based in part on the airlines' willingness to equip aircraft with the required technologies, and it is likely that future air traffic controllers (ATCos) will have to manage airspaces that have more aircraft than presently allowed in a given sector. Moreover, these aircraft will vary in terms of the type of tools for communication, navigation, and conflict resolution currently onboard.

Therefore, it is essential that air traffic control displays be configured to provide information about the characteristics of individual and groups of aircraft currently in a given sector. However, relatively little attention is being paid to the design of displays and symbols for NextGen ATCos. This is perhaps because strategies for the allocation of roles and responsibilities are still being worked out (e.g., Strybel et al., 2010; Prevot, Homola, Martin, Mercer, \& Cabrall, 2012). In fact, most simulations of NextGen operating concepts, function allocation, and impact of automation are based on symbology that is either derived from current day ATCo symbology or determined for each simulation on a case-bycase basis. In this paper, we report on a preliminary study designed to investigate the appropriate symbology for NextGen air traffic control displays that would be used in mixed-equipage airspaces.

Symbols and icons are commonly used in air traffic management systems to convey specific pieces of traffic information, such as location/position, direction of travel, and alert level. This information can be represented by a number of visual features or characteristics, including shape, color, and size, to name a few. Air traffic controllers must be able to perceive and interpret the symbols on their radar displays, to update the traffic picture and ensure the safe and efficient management of aircraft in their sectors. However, there is a lack of standardization within and across air traffic management systems concerning the design and implementation of the appropriate symbols to use in order to convey important traffic information. This lack of standardization can lead to confusion, misinterpretation, and, ultimately, operational errors. It is imperative, therefore, to determine early on which symbols and symbol features are most intuitive and easy to learn for effective air traffic management.

Chandra, Zuschlag, Helleberg, and Estes (2009) recently conducted a web-based study assessing pilots' ability to learn and remember traffic symbols that may be shown on Cockpit Displays of Traffic Information (CDTI). Specifically, they evaluated three aspects deemed important for symbol design: Intuitiveness, ease of learning, and ease of remembering. Chandra et al. found that the best symbols had clear direction indicators (e.g., a leading line or pointed head) and used conventional red or yellow colors to indicate alert level. McDougall, Tyrer, and Folkard (2006) examined participants' reaction time (RT) and accuracy to detecting pre-specified target symbols and reported that simple symbols conveying two pieces of information were better than complex symbols conveying three of more pieces of information (see also Xing, 2007; Xing \& Manning, 2005).

Evaluations of symbology and symbol features for air traffic control have been focused on reducing the complexity of the displays. For example, Ahlstrom, Rubinstein, Siegel, Mogford, and Manning (2001) examined four display enhancements for reducing sector complexity caused by Special Use Airspace, weather, reliability of radio and radar coverage, and number of transitioning aircraft. Ahlstrom et al. found that ATCos favored color and graphical enhancements for reducing complexity. However, Yuditsky et al. (2002) evaluated the application of one enhancement, color-coding, for the impacts on performance in an air traffic management simulation. Although color-coding did improve performance when the color-coding was tested individually, no benefit was found when the enhancements were combined in the context of air traffic control. 
It is important to note that none of the aforementioned studies examined combinations of symbols and symbol features for their effectiveness in reducing complexity in future mixed-equipage airspaces. Just what features of symbols might best represent Data-Comm equipped aircraft, for example, are, as yet, unknown. The advent of NextGen concepts and technologies in the NAS opens up a unique new opportunity to re-evaluate current day air traffic symbology and propose potentially new symbol features and designs.

We report on an initial investigation of symbol features to determine the intuitiveness of air traffic symbology. Here, we systematically explored whether certain symbol features are best used to represent specific pieces of traffic and aircraft status information.

\section{Methods}

\section{Survey 1}

Participants. Twenty-one (18 males; age range $=23-67$ years; mean age $=32$ years) participants completed an online survey, which took approximately 30 minutes to complete. Six of the participants reported no previous air traffic control experience. Another four reported having at least 10 years of air traffic control experience. The remaining participants reported having some experience with air traffic control simulation software.

Design and Procedure. Participants followed a link to an online questionnaire that was e-mailed to them as an invitation to take part in the study (surveymonkey.com). The questionnaire included demographic questions concerning gender, age, ethnicity, air traffic control experience, flight training, and gaming experience. Before beginning the main part of the questionnaire, participants were given some basic background information about air traffic management symbology and they were informed of the purpose of the study. The participants were also given information concerning the meaning of aircraft ownership, data link equipage, altitude, and heading. A sample question was provided before participants began completing the main survey questions.

Table 1. A small subset of the 96 symbols used in the present study. Symbols shown are unfilled and white.

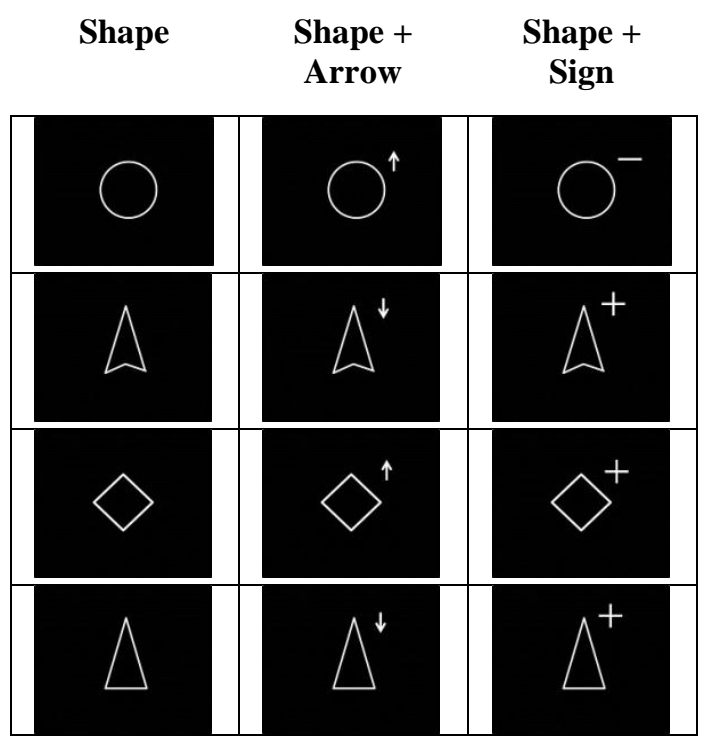

Each question in the survey asked participants to indicate what information they thought could be conveyed by the symbol displayed. The symbols varied in shape and color, and whether filled or open. Four colors were tested, blue, green, white, and yellow. The color red was not used here because of its strong association with conflicts or danger (Xing, 2006). Twelve symbols were evaluated, shown in Table 1 . These consisted of four shapes presented alone and with arrows or $+/$ - signs to the upper right of the symbol. The shapes were selected based on current usage in air-traffic displays and cockpit displays of traffic information. The addition of arrows was examined because they are commonly used in pilot displays to convey changes in altitude. The use of +/- signs has not been explored previously. Lastly, all symbols were presented as filled or unfilled. The combinations of shape, color, and filled/unfilled features created 96 different symbols that were presented to each participant in random order.

Each symbol was presented one at a time on the computer screen, against a black background. Participants could select one or more of any of the following information options for each symbol. These were categorized as follows:

- Equipage: Equipped with Data Comm or Unequipped with Data Comm

- Trajectory: Heading, Climbing, or Descending

- Ownership: Owned by your Sector or Unowned by your sector (see Figure 1).

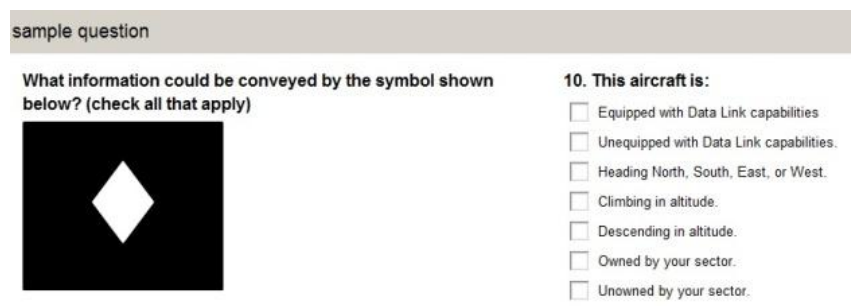

Prev Nert

Figure 1. Sample question layout for Survey 1.

\section{Results and Discussion}

The percentage of responses falling into each of the three information categories was determined for each symbol and symbol feature. Chi-square contingency tests with Symbol Feature and Information Category as the two variables were conducted to determine which symbol features were related to equipage, heading, and ownership. Chi-square tests were run for Fill of the aircraft (AC) Symbol, Color of the AC Symbol, and Shape of the AC Symbol.

The chi-square test for Symbol Fill was nonsignficant. It is important to note that ownership was selected for approximately $91 \%$ of both filled and unfilled symbols. A closer look at the type of information participants associated with filled versus unfilled symbols shows that regardless of symbol shape or color, unfilled symbols were designated most often as unowned, and filled symbols as owned as shown in Figure 2.

The chi-square test on Symbol Color also revealed no significant relationship between Symbol Color and Information Category. In effect, none of the colors was seen as related to a specific information category. The responses in each category were equally divided among the four colors used. 


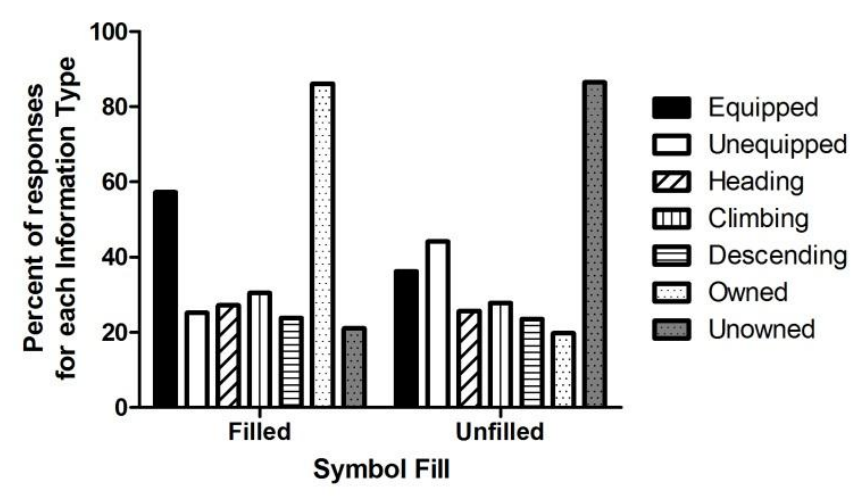

Figure 2. The percentage of responses for each Information Type as a function of Fill of the aircraft symbol.

The chi-square test on Symbol Shape revealed a significant relationship with Information Category, $\chi^{2}(22)=212.5, p<.001$. Each information category was therefore evaluated separately, with one-way chi-square tests. Differences in percentage of ownership responses were nonsignificant. However, the percentage of equipage responses were significantly different, $\chi^{2}(11)=94.3, p<.05$, as shown in Figure 3.

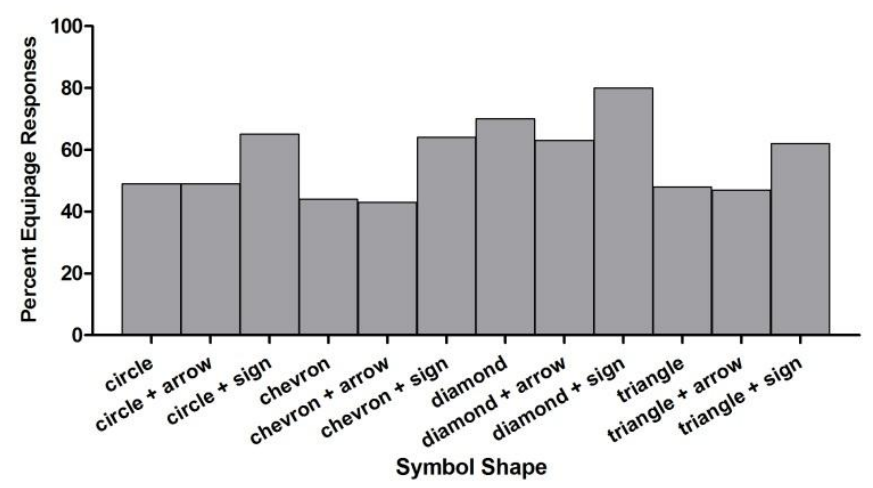

Figure 3. Percent of Equipage Responses as a Function of Symbol Shape.

The percentage of trajectory responses were also significantly different, $\chi^{2}(11)=270.0, p<.001$, as shown in Figure 4.

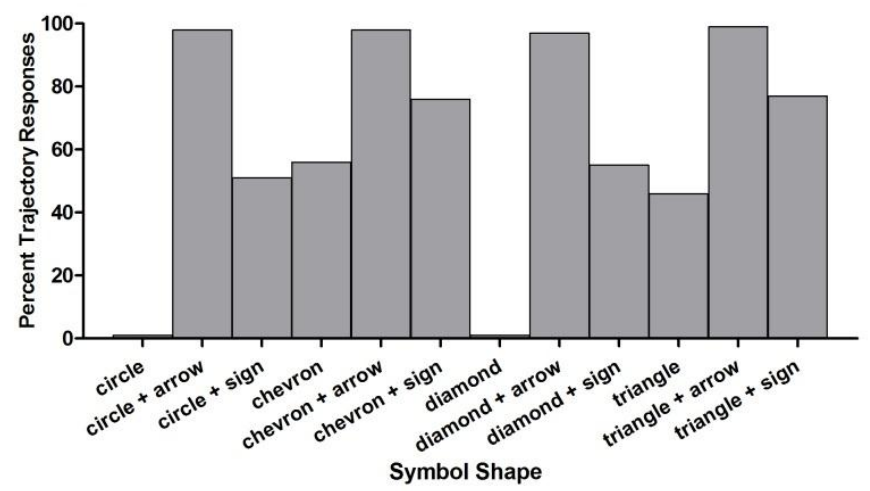

Figure 4. Percentage of Trajectory Responses as Function of Symbol Shape and Additional Features.
Figures 5 and 6 provide a closer look at the information types that comprised Trajectory responses (Altitude Change: Climbing or Descending, and Heading) based on the Symbols that included an Extra Feature (Up/Down Arrow or $+/$ - Sign).

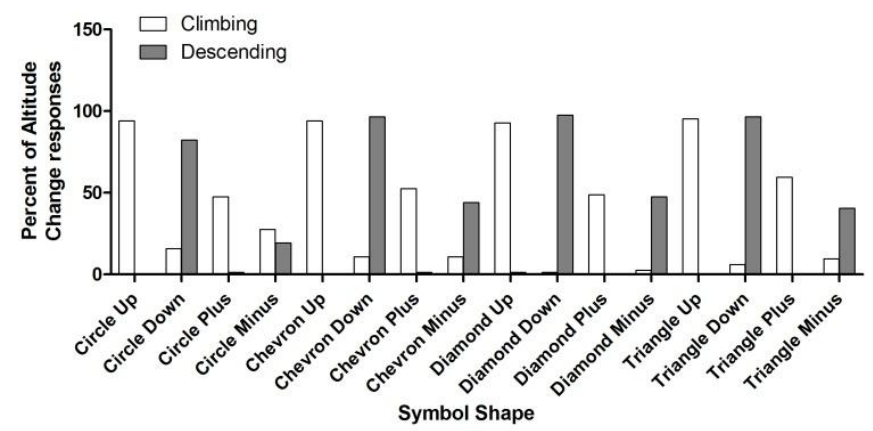

Figure 5. Percentage of Altitude Change Responses as a Function of Symbols with Extra Arrows and Signs.

From Figure 5, it is clear that any symbol with an Extra up arrow is associated with increasing altitude changes, while down arrows are associated with decreasing altitude changes.

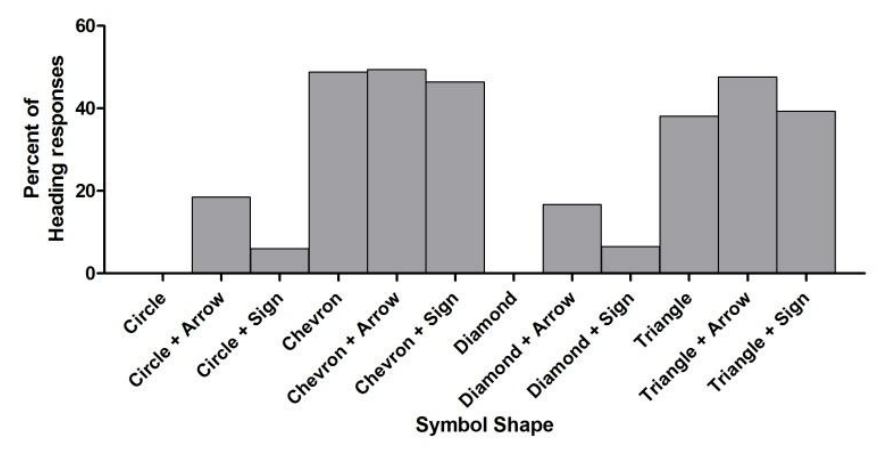

Figure 6. Percentage of Heading Responses as Function of Symbol Shape.

From Figure 6, it is apparent that neither the circle or diamond symbol shapes were preferred for conveying heading information. This makes sense given that circles and diamonds do not provide clear directional information, while chevrons and triangles do. This finding is consistent with those of Chandra et al. (2009) showing that symbols with leading lines or pointed head were the best indicators of heading information.

Trajectory (i.e., altitude and heading) is a well-developed concept in current day air traffic displays, but these are typically portrayed by text. The results of Survey 1 suggest that trajectory (especially altitude change) can be represented with an up/down arrow accompanying the aircraft symbol. Here, participants associated any up arrow accompanying the symbol with increasing altitude and any down arrow accompanying the symbol with decreasing altitude. Note that altitude changes or transitions were identified by Ahlstrom et al. (2001) as being a contributing factor to sector complexity.

\section{Methods}

\section{Survey 2}

Participants. Twenty-one (19 males; age range $=21-65$ years; mean age $=30$ years) participants completed an online survey, which took approximately 15 minutes to complete. The participants who completed Survey 2 were invited due to their reported experience with air traffic control displays. The 
goal of Survey 2 was to explore how those who are familiar with air traffic management and air traffic displays would use symbols to represent certain types of air traffic information. Additionally, Survey 2 aimed to further explore more specific NextGen concepts and tools.

Instead of having participants select the information that could be represented by given symbols, Survey 2 required participants to select a single symbol feature to represent a given Information Type as shown in Figure 7. The Symbol Features included in Survey 2 were: Fill of AC symbol, Brightness of AC symbol, Color of AC symbol, Shape of AC symbol, Shape of data tag symbol, or Text in data tag. The Information Types were: Altitude, Spacing Equipage (an AC's equipage with Airborne Self-Spacing Tools), Data Comm Equipage, Flight Rules (Autonomous Flight Rules, AFR; Instrument Flight Rules, IFR; or Visual Flight Rules, VFR), Flight Type (Arrival, ARR; Departure, DEP; or Overflight, OVR).

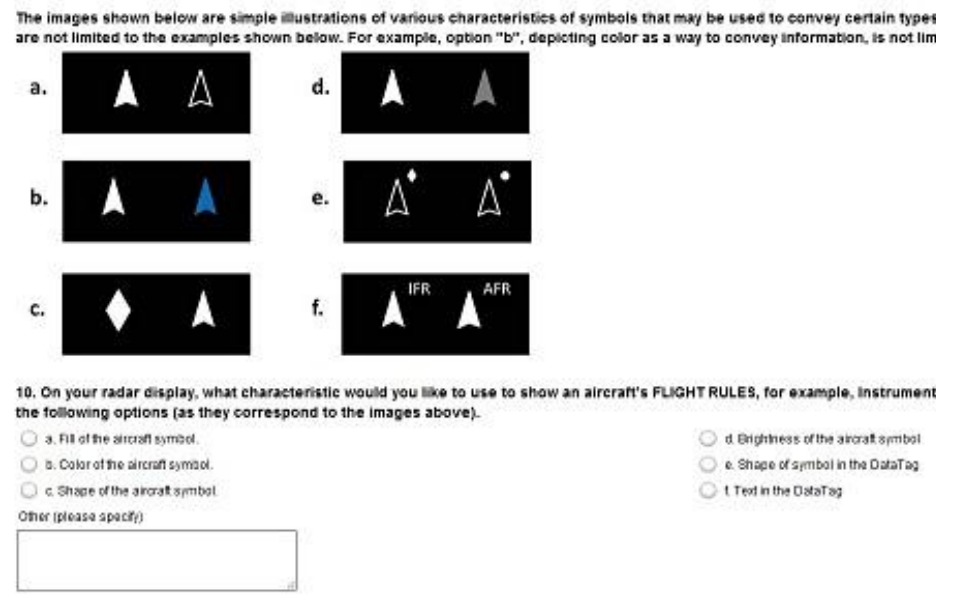

Figure 7. Sample question layout for Survey 2.

\section{Results and Discussion}

The percentage of responses for each symbol feature was collected for each of the six information types (see Figure 8). Separate Chi-square tests were conducted for five of the six Information Types: Flight Rules, Spacing, Flight Type, DataComm, and Ownership. A Chi-Square test was not conducted for Altitude because all but one participant selected text in the data tag to represent altitude.

The Chi-square test revealed that Flight Rules (AFR, VFR, or IFR) were best represented by the color of the AC symbol, with participants choosing this feature $47 \%$ of the time, $\chi^{2}(5)=16.43, p=.01$. Participants chose Text in the data tag to convey an AC's Spacing capabilities $52 \%$ of the time, $\chi^{2}(5)=21.00, p=.01$. DataComm equipage was best conveyed by the Shape of the data tag symbol, being selected $52 \%$ of the time, $\chi^{2}(5)=21.00, p=.01$. The fill of the AC symbol best conveyed Ownership (47\%), $\chi^{2}(5)=16.43, p=.01$. No preference was shown, however, to represent flight type (ARR, DEP, or OVR).

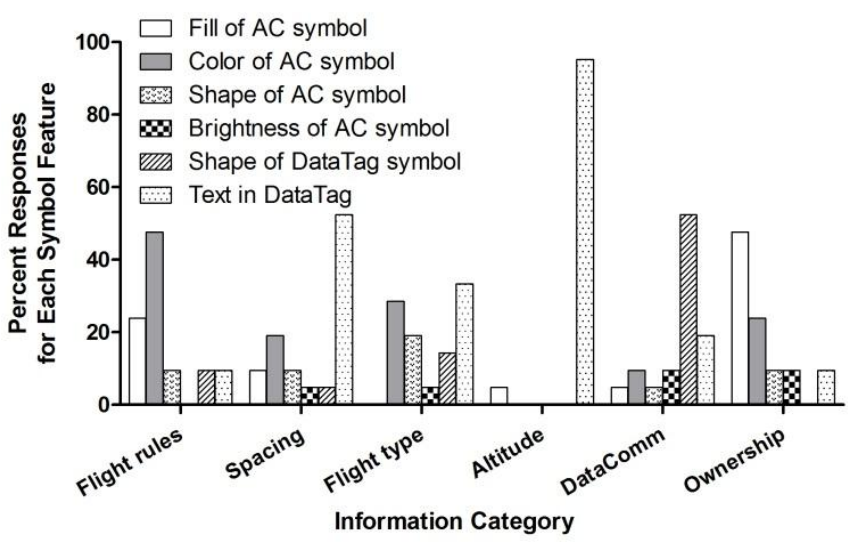

Figure 8. Percent of Responses for Each Symbol Feature as a Function of Information Category.

The results of Survey 2 illustrated that experienced air traffic controllers and those experienced with air traffic displays had clear preferences for symbol features used to convey certain types of air traffic information. Follow-up subjective questions revealed that all of the participants' choices were based on prior experience. It is not surprising that the use of text in the data tag was almost unanimously chosen to represent altitude, because this has been a longstanding convention in air traffic management. Data Comm equipage appeared to be well conveyed by the shape of the data tag symbol, while spacing equipage appeared to be best represented via text in the data tag. The AC's flight rules were best represented with the color of the AC symbol, and Ownership was best conveyed by the fill of the AC symbol.

\section{GENERAL DISCUSSION}

The present study sought to explore the visual features that would best communicate important air-traffic information. The results of the two surveys reported here indicate that the convention for symbolizing ownership and altitude, concepts that have been well established in current day displays of air traffic are clearly associated with AC symbol fill and data tag text, respectively. In fact, a number of participants subjectively reported that text was the only way to appropriately convey altitude information. With respect to ownership, participants associated any filled symbol with owned AC and any unfilled symbol with unowned AC regardless of color or shape.

Information regarding NextGen equipage, however, is not found on many current day air traffic displays. The results of Survey 1 and 2 both suggest that the shape of the symbol could be the best way to represent an AC's Data Comm equipage. While the results of Survey 1 suggested that symbol color was a poor indicator of equipage, trajectory, and ownership, Survey 2 revealed that an AC's flight rules could be efficiently conveyed by the color of the AC symbol. It is interesting to note that an AC's spacing capabilities appeared to be best conveyed via text in the data tag. The fact that a clear preference was found here warrants further exploration with the caution that this additional text may increase the clutter in the data tag and air traffic display overall. As such, it 
may be worthwhile to explore alternative ways to communicate spacing equipage.

Taken together, the results of the present study suggest that certain symbol features should be reserved to convey specific types of air traffic information, as they not only follow convention, but also because they are intuitive for naïve air traffic controllers. The fact that participants in Survey 2 displayed clear preferences for Data Comm and spacing equipage is promising, given the inevitable advent of NextGen concepts and tools. Implementing air traffic symbols that are intuitive, easy-to-learn and recognize, and follow previously-developed conventions will not only minimize the time spent training ATCos on new air traffic concepts and tools, but it can also aid in the efficient management of air traffic. This initial survey study is the first in a series of experiments designed to examine symbology and visual features for current day and NextGen air traffic management concepts and technologies.

\section{References}

Ahlstrom, U., Rubinstein, J., Siegel, S., Mogford, R., Manning, C. (2001). Display concepts for en route air traffic control (Report No. DOT/FAA/CTTN01/06). Springfield, VA: National Technical Information Service.

Chandra, D., Zuschlag, M., Helleberg, J., \& Estes, S. (2009). Symbols for cockpit displays of traffic information. Proceedings of the 28th Digital Avionics Systems Conference October 25-29, 2009, Orlando, FL.

Erzberger, H. (2004). Transforming the NAS: The next generation air traffic control system (Report No. NASA/TP-2004-212828). Moffett Field, CA: NASA Center for AeroSpace Information.

Federal Aviation Administration (2011). FAA's NextGen Implementation Plan, March 2011. Federal Aviation Administration.

McDougall, S., Tyrer, V., \& Folkard, S. (2006). Searching for signs, symbols, and icons: Effects of time of day, visual complexity, and grouping. Journal of Experimental Psychology: Applied, 12, 118-128.

Prevot, T., Homola, J. R., Martin, L. H., Mercer, J. S., \& Cabrall, C. D. (2012). Toward automated air traffic control - Investigating a fundamental paradigm shift in human/systems interaction. International Journal of Human-Computer Interaction, 28, 77-98.

Strybel, T.Z., Vu, K.-P. L., Bacon, L. P, Kraut, J., Minakata, K., Battiste, V. \& Johnson, W. (2010). Diagnosticity of an Online Query Technique for Measuring Pilot Situation Awareness in NextGen. Proceedings of the 29th Digital Avionics Systems Conference (pp. 4.B.11-4.B.1-12). Salt Lake City, Utah: IEEE.

Xing, J. (2006). Color analysis in air traffic control displays, part I. Radar displays (Report No. DOT/FAA/AM06/22). Springfield, VA: National Technical Information Service.

Xing, J. (2007). Information complexity in air traffic control displays (Report No. DOT/FAA/AM-07/26). Springfield, VA: National Technical Information Service.
Xing, J., \& Manning, C. A. (2005). Complexity and automation displays of air traffic control: Literature review and analysis (Report No. DOT/FAA/AM05/4). Springfield, VA: National Technical Information Service.

Yuditsky, T., Sollenberger, R. L., Della Rocco, P. S., \& Manning, C. (2002). Application of color to reduce complexity in air traffic control (Report No. DOT/FAA/CT-TN03/01. Springfield, VA: National Technical Information Service.

This research was supported by the NASA cooperative agreement NNX09AU66A, Group 5 University Research Center: Center for Human Factors in Advanced Aeronautics Technologies (Ms. Brenda Collins, Technical Monitor). 